\title{
Ueber das Chinosol, sein Verhalten im Thierkörper und über die Bildung gepaarter Glukuronsäuren.
}

\author{
Von
}

Carl Brahm, Apotheker.

(Aus der chemischen Abtheilung des physiologischen Instituts in Berlin.)

(Der Redaction zugegangen am 12. August 1899.)

Vor 2 Jahren erhielt Herr Prof. Thierfelder durch Herrn Regierungsrath Jacobj aus dem Kaiserlichen Gesundheitsamt Krystalle, die sich aus dem Harn von Thieren nach Eingabe von Chinosol spontan ausgeschieden hatten. Prof. Thierfelder betraute mich mit der näheren Untersuchung dieser Erscheinung. Die Ergebnisse sollen im Folgenden mitgetheilt werden.

Das Chinosol ist ein in letzter Zeit vielfach als Antiseptikum, Antipyretikum und Desinficiens angepriesenes Mittel, dessen Herstellung durch deutsches Reichspatent (D. R. P. 88520) gesichert ist. Die Fabrik bezeichnet dasselbe als chinophenylschwefelsaures Kalium und auch als oxychinolinsulfosaures Kalium. Die Darstellung geschieht nach den Angaben der Patentschrift durch 10 - bis 12 stündiges Kochen von 2 Molekiilen o-Oxychinolin in alkoholischer Lösung mit 1 Molekül Kaliumpyrosulfat am Rückflusskühler unter Umrühren. Der Process soll nach folgender Gleichung verlaufen:

$$
2 \mathrm{C}_{9} \mathrm{H}_{6} \mathrm{NOH}+\mathrm{K}_{2} \mathrm{~S}_{2} \mathrm{O}_{7}=2 \mathrm{C}_{9} \mathrm{H}_{6} \mathrm{NO} \mathrm{SO}_{3} \mathrm{~K}+\mathrm{H}_{2} \mathrm{O} \text {. }
$$

Das Chinosol stellt ein krystallinisches, schwefelgelbes Pulver dar, in Wasser in jedem Verhältniss löslich. Die Lösung reagirt sauer und gibt mit Eisenchlorid eine schwarzgrüne Fällung. Durch Sodalösung trübt sie sich und erstarrt zu einem Krystallbrei von o-Oxychinolin. Auf dieses Verhalten, welches 
sich mit der Auffassung des Körpers als Salz einer Sulfosäure oder Aetherschwefelsäure nicht verträgt, werde ich noch zurückkommen.

Das für meine Untersuchungen nöthige Material wurde mir von der Chinosolfabrik Franz Fritzsche \& Co. in Hamburg bereitwilligst zur Verfügung gestellt.

Als Versuchsthiere dienten Hunde und Kaninchen. Einem mittelgrossen Hunde von 17,5 kg Körpergewicht gab ich Anfangs mit der Schlundsonde $10 \mathrm{~g}$ Chinosol in wässeriger Lösung in den Magen. Da jedoch nach ungefähr 10 Minuten Erbrechen eintrat, ging ich mit der Dose auf $5 \mathrm{~g}$ hinunter, jedoch erbrach auch hierbei das Thier häufig genug. Die Kaninchen erhielten die Substanz ebenfalls in wässeriger Lösung mit der Schlundsonde anfänglich in Dosen von $5 \mathrm{~g}$. Da indessen die Fresslust abnahm und nach 7 bis spätestens 11 Tagen der Tod eintrat, verringerte ich die tägliche Dosis auf $1 \mathrm{~g}$. Hierbei zeigten sich keine unangenehmen. Folgen. Eines der so behandelten Thiere lebte 47 Tage, ging aber am 48. auf eine Gabe von $5 \mathrm{~g}$ nach 2 Stunden zu Grunde. Nicht nur der Hunde-, auch der Kaninchenharn zeigte nach Zufuhr von Chinosol stets saure Reaction. Gelegentlich schieden sich aus Kaninchenharn schon nach längerem Stehen grünlichweisse Krystalle von schön ausgebildeter Form und ansehnlicher Grösse (bis $\mathrm{zu}{ }_{.}^{1 / 2} \mathrm{~cm}$.) in grösserer oder geringerer Menge aus, und zwar wurde das stets beobachtet, wenn der Harn in Folge wasserarmer und "saurer» Nahrung (Haferschrot) von vornherein sauer und concentrirt war und das Thier eine grose Chinosoldosis bekommen hatte. Die Erscheinung blieb dagegen aus bei wasserreicher und «alkalischer» Nahrung (Grünfutter) und bei kleiner Chinosolgabe. Im Hundeharn konnte ich die spontane Abscheidung von Krystallen nur einmal beobachten. Die Krystalle waren stark lichtbrechende Doppelpyramiden von quadratischem Habitus, ganz wie Anatas aussehend.

Die Harne drehten regelmässig die Ebene des polarisirten Lichtes nach links, stärker oder schwächer, je nach Concentration und Chinosolgabe; ferner vermochten sie bei Gegenwart von Alkali viel Kupferoxyd in Lösung zu halten. Eine Reduc- 
tion zu Kupferoxydul trat beim Erwärmen nicht ein, wohl aber kam es zu einer solchen, wenn der Harn vorher mit verdünnter Salzsäure längere Zeit erhitzt worden war. Diese beiden Eigenschaften des Harns, die Ablenkung der Ebene des polarisirten Lichtes nach links und die Einwirkung auf Fehling'sche Lösung nach dem Erwärmen mit verdünnter Mineralsäure sind charakteristisch für eine Gruppe von Stoffwechselprodukten, die unter dem Namen gepaarte Glukuronsäuren zusammengefasst werden. Es lag nahe daran zu denken, dass der Harn nach Eingabe von Chinosol eine solche Verbindung enthielt.

Isolirung und Eigenschaften der linksdrehenden und nach dem Kochen mit Säure reducirenden Substanz.

Die spontan ausgeschiedenen Krystalle trennte ich durch Filtration von dem Harne und krystallisirte sie mehrfach aus heissem Wasser um. Um die in der Harnflüssigkeit noch gelöste Substanz zu isoliren, verfuhr ich zunächst nach einer Methode, welche, zuerst von Muskulus und von Mering ${ }^{1}$ ) zur Gewinnung der Urochloralsäure angegeben, später auch von $\mathrm{Kül} \mathrm{z}^{2}$ ) benützt, auf der Löslichkeit mancher gepaarter Glukuronsäuren in Aetheralkohol beruht. Zu dem Zweck dampfte ich den Harn von mehreren Tagen zum dünnen Syrup ein und schüttelte wiederholt mit einer Mischung von 1 Liter Aether, $500 \mathrm{ccm}$. Alkohol, $15 \mathrm{ccm}$. Wasser und $15 \mathrm{ccm}$. concentrirter Schwefelsäure aus. Von den vereinigten ätherisch-alkoholischen Filtraten destillirte ich den Aetheralkohol ab, neutralisirte den in wenig Wasser aufgenommenen Rückstand genau mit Barytwasser, filtrirte vom schwefelsauren Baryt $a b$, fällte die Flüssigkeit mit Bleizucker und das vom entstandenen Niederschlag befreite Filtrat vorsichtig mit Bleiessig. Den mit heissem Wasser gut ausgewaschenen basischen Bleiniederschlag zerlegte ich mit Schwefelwasserstoff. Die vom überschüssigen Schwefelwasserstoff befreite Flüssigkeit gab keine Reaction auf gepaarte Glukuronsäure. Die Verbindung war also nach dieser Methode aus dem

1) Ber. d. d. chem. Gesellsch., 8, 662.

2) Zeitschr. f. Biol., 27, 277. 
Harn nicht zu isoliren, und zwar, wie sich später zeigte, deshalb nicht, weil sie in Aetheralkohol unlöslich ist. Ich fällte nun den frischen Harn direkt mit neutralem und das Filtrat mit basischem Bleiacetat aus. Dieser letztere Niederschlag wurde mit Wasser gewaschen und mit Schwefelwasserstoff zerlegt. Aus der mit Thierkohle entfärbten Flüssigkeit schieden sich nach entsprechender Concentration beim Erkalten grosse wasserhelle Krystalle aus, die sich als völlig identisch mit den spontan ausgefallenen erwiesen. Aus Kaninchen- und Hundeharn wurde ganz die gleiche Substanz erhalten.

Die Krystalle lösen sich sehr schwer in kaltem, leicht in heissem Wasser. $100 \mathrm{ccm}$. einer bei $15^{\circ}$ gesättigten Lösung enthielten $0,12275 \mathrm{~g}$, entsprechend einer Löslichkeit von $1 \mathrm{Thl}$. in 815 Thl. Wasser von $15^{\circ}$. Die Löslichkeit wird bedeutend erhöht durch Anwesenheit von Mineralsäuren, nicht dagegen durch Essigsäure. Die Substanz ist unlöslich in Alkohol, Aether, Chloroform u. s. w., leicht löslich in Alkali. Der Schmelzpunkt ist nicht scharf. Bei $102-105^{\circ}$ tritt Gelbfärbung ein, bei $151^{0}$ Zersetzung. Beim Erhitzen im trockenen Reagensglas bläht sich die Substanz stark auf, verkohlt und scheidet ein gelbes krystallinisches Sublimat ab. Die wässerigen Lösungen reagiren stark sauer, sie reduciren Fehling'sche Fülüssigkeit nicht direkt, sondern erst nach längerem Kochen mit .verdünnter Mineralsäure. Die Untersuchung im Polarisationsapparat lässt sich wegen der Schwerlöslichkeit nicht ausführen.

Die Krystalle enthielten $\mathrm{C}, \mathrm{H}, \mathrm{N}$ und $\mathrm{O}$, aber keinen $\mathrm{S}$. Für die quantitativen Bestimmungen wurden sie fein zerrieben und im Vacuum über Schwefelsäure bis zur Gewichtsconstanz getrocknet. Hierbei verlor die lufttrockene Substanz 10,05\% Wasser $(0,3738 \mathrm{~g}$ verloren $0,0376 \mathrm{~g}$ Wasser).

Die Stickstoffbestimmung führte ich nach der von M. Krüger ${ }^{1}$ ) angegebenen Modification (Benutzung von Kaliumbichromat) der Kjeldahl'schen Methode, welche auch bei den Chinolinderivaten völlige Gewähr für die Ueberführung des $\mathrm{N}$ in $\mathrm{NH}_{3}$ bietet, aus. Sie ergab folgende Resultate:

1) Ber. d. d. chem. Gesellsch., 27, 609. 
1. $0,12525 \mathrm{~g}$ Substanz verbrauchten $3,8 \mathrm{ccm}$. 1/10 N-Säure.

2. $0,2143 \check{g}$ g Substanz verbrauchten $6,6 \mathrm{ccm}$. 1/10 N-Säure.

Gefunden:

1. $4,24 \% \mathrm{~N}$

2. $4,31 \% \mathrm{~N}$.
Berechnet für $\mathrm{C}_{15} \mathrm{H}_{15} \mathrm{NO}_{7}$ : $4,36 \% \mathrm{~N}$.

Obgleich auf die Bestimmungen des Kohlen- und Wasserstoffs grosse Sorgfalt verwandt und mit der Methode vielfach gewechselt wurde, gelang es mir nicht, untereinander stimmende Resultate $\mathrm{zu}$. erhalten. Der Grund lag jedenfalls in der ausserordentlichen Schwerverbrennlichkeit des schon bei geringer Hitze an der Rohrwand sich niederschlagenden Sublimats.

In der Hoffnung, durch die Analyse der Salze besseren Aufschluss über die Zusammensetzung der Säure zu gewinnen, stellte ich zunächst das

Kalisalz

dar. Dasselbe liess sich leicht erhalten durch Umlegen der durch Kochen der wässerigen Lösung der freien Säure mit Baryumkarbonat hergestellten Barytsalzlösung mit Kaliumsulfat. Aus der vom Baryumsulfat abfiltrirten Flüssigkeit schied sich nach entsprechender Concentration die Kaliverbindung in schönen wasserhellen Pyramiden aus. Auf Alkoholzusatz entstand in der Mutterlauge noch eine weitere Krystallisation. Die Krystalle sind sehr leicht löslich in kaltem Wasser, unlöslich in absolutem Alkohol. Zur Analyse diente das im Vacuum über Schwefelsäure bis zum constanten Gewicht getrocknete Salz. Die Verbrennung führte ich im geschlossenen Rohr mit Bleichromat und nachträglichem Sauerstoffdurchleiten, die Stickstoffbestimmung wieder nach M. Krüger, die Kalibestimmung durch Abrauchen mit concentrirter Schwefelsäure in der Platinschaale aus.

1. 0,2012 g Substanz lieferten $0,3543 \mathrm{~g} \mathrm{CO}_{\mathbf{z}}$ und $0,0896 \mathrm{~g} \mathrm{H}_{\mathbf{g}} \mathrm{O}$.

2. $0,2079 \mathrm{~g}$ Substanz lieferten $0,3656 \mathrm{~g} \mathrm{CO}_{2}$ und $0,0964 \mathrm{~g} \mathrm{H}_{2} \mathrm{O}$.

3. $0,2874 \mathrm{~g}$ Substanz verbrauchten $7,15 \mathrm{ccm}$. $1 / 10 \mathrm{~N}$-Säure.

4. $0,28935 \mathrm{~g}$ Substanz verbrauchten $7,35 \mathrm{ccm}$. 1/10 N-Säure.

5. $0,2086 \mathrm{~g}$ Substanz verbrauchten $5,4 \mathrm{ccm}$. 1/10 N-Säure.

6. $0,3726 \mathrm{~g}$ Substanz lieferten $0,08165 \mathrm{~g} \mathrm{~K}_{2} \mathrm{SO}_{4}$.

7. $0,3334 \mathrm{~g}$ Substanz lieferten $0,0736 \mathrm{~g} \mathrm{~K}_{2} \mathrm{SO}_{4}$.

$\begin{array}{cccccccccc} & 1 & 2 & 3 & 4 & 5 & 6 & 7 & \text { Mittel } & \mathrm{C}_{15} \mathrm{H}_{14} \mathrm{NO}_{7} \mathrm{~K} \\ \mathrm{C} & 48,03 & 47,97 & - & - & - & - & - & 48,00 & 47,74 \\ \mathrm{H} & 4,95 & 5,15 & - & - & - & - & - & 5,05 & 4,45 \\ \mathrm{~N} & - & - & 3,48 & 3,55 & 3,62 & - & - & 3,55 & 3,71 \\ \mathrm{~K} & - & - & - & - & - & 9,82 & 9,89 & 9,85 & 10,03\end{array}$


Baryumsalz.

Zur Darstellung wurde die wässerige Lösung der freien Säure mit Baryumkarbonat gekocht, filtrirt und eingedampft, der Rückstand in Wasser aufgenommen. Beim Verdunsten schieden sich schneeweisse verfilzte Nadeln des Barytsalzes aus. Dieselben sind in Wasser leicht löslich, in Alkohol unlöslich und erhalten, im Vacuum über Schwefelsäure getrocknet, soviel Baryum, wie die Formel $\left(\mathrm{C}_{15} \mathrm{H}_{14} \mathrm{NO}_{7}\right)_{2} \mathrm{Ba}+2 \mathrm{H}_{2} \mathrm{O}$ verlangt.

1. $0,1526 \mathrm{~g}$ Substanz lieferten $0,0442 \mathrm{~g} \mathrm{Ba} \mathrm{SO}_{4}$.

2. 0,2327 g Substanz lieferten $0,0676 \mathrm{~g} \mathrm{Ba} \mathrm{SO}_{4}$.

Gefunden:

1. $17,03 \% \mathrm{Ba}$

2. $17,06 \% \mathrm{Ba}$.
Berechnet:

$16,85 \% \mathrm{Ba}$.

\section{Strontiumsalz.}

Das in derselben Weise hergestellte Strontiumsalz verhielt sich in jeder Beziehung wie das Baryumsalz.

1. $0,2108 \mathrm{~g}$ Substanz lieferten $0,0518 \mathrm{~g} \mathrm{Sr} \mathrm{SO}_{4}$.

2. $0,09105 \mathrm{~g}$ Substanz lieferten $0,02195 \mathrm{~g} \mathrm{SrSO}_{4}$.

\section{Gefunden:}

1. $11,71 \% \mathrm{Sr}$

2. $11,49 \% \mathrm{Sr}$.

Berechnet: $11,78 \% \mathrm{Sr}$.

\section{Cadmiumsalz.}

Die Herstellung geschah durch Umlegen des Baryumsalzes mit Cadmiumsulfat. Es krystallisirt in feinen weissen Nadeln, ist in Wasser leicht löslich und hat ebenso, wie das Baryumund Strontiumsalz, stark elektrische Eigenschaften. Die Analyse des über Schwefelsäure im Vacuum getrockneten Salzes stimmte $\mathrm{zu}$ der Formel $\left(\mathrm{C}_{15} \mathrm{H}_{14} \mathrm{NO}_{7}\right)_{2} \mathrm{Cd}$.

1. $0,2013 \mathrm{~g}$ Substanz lieferten $0,0392 \mathrm{~g}$ Cd S.

2. $0,1906 \mathrm{~g}$ Substanz lieferten 0,0355 g Cd S.

Gefunden:

1. $15,14 \% \mathrm{Cd}$.

2. $14,49 \%$ Cid.
Berechnet:

$14,89 \%$ Cd.

Auch das Zink-, das Blei- und das Nickelsalz liessen sich leicht in schönen Krystallen erhalten. Sie wurden nicht analysirt.

Nach den mitgetheilten Analysen der Salze und den Stickstoffbestimmungen der freien Säure kommt dieser die 
Formel $\mathrm{C}_{15} \mathrm{H}_{15} \mathrm{NO}_{7}$ zu. Sie krystallisirt mit 2 Molekülen Krystallwasser, welches im Vacuum abgegeben wird, denn die lufttrocknen Krystalle verloren im Vacuum, wie oben angegeben, 10,05\% Wasser (berechnet 10,08\%).

\section{Specifische Drehung des Kalisalzes.}

Die Bestimmung geschah in einem Lippich'schen Halbschattenapparat. Das Salz war im Vacuum getrocknet. Zur Bestimmung des Nullpunktes und des Drehungswinkels wurden aus einer ganzen Reihe von Einzelbestimmungen die Mittel genommen.

\begin{tabular}{c|c|c|c|c|c|c}
\hline \hline Nr. & $\begin{array}{c}\text { Substanz in } \\
100 \mathrm{ccm} .\end{array}$ & $\begin{array}{c}\text { Substanz in } \\
100 \mathrm{~g}\end{array}$ & $\begin{array}{c}\text { Sehalt an } \\
\text { Gewicht } \\
\text { bei } 18^{\circ}\end{array}$ & $\begin{array}{c}\text { Specifisches } \\
\text { länge } \\
\text { in } \\
\text { dm. }\end{array}$ & $\begin{array}{c}\text { Rohr- } \\
\text { Winkel- } \\
\text { drehung } \\
\text { bei } 18^{0}\end{array}$ & $\begin{array}{c}\text { Specifische } \\
\text { Drehung } \\
(\alpha) \mathrm{D}\end{array}$ \\
\hline 1 & 4,2285 & 4,1559 & 1,0175 & 2 & $-7,1$ & 83,83 \\
\hline 2 & 3,7276 & 3,6719 & 1,01519 & 2 & $-6,1$ & 81,82 \\
\hline 3 & 1,8674 & 1,8530 & 1,0078 & 2 & $-2,86$ & 76,59
\end{tabular}

Wie sich aus der Tabelle ergibt, nimmt die specifische Drehung mit abrehmender Concentration der Lösung ab.

$$
\text { Spaltungsprodukte. }
$$

Die gefundene Zusammensetzung der Substanz stimmt sehr gut zu der Annahme, dass eine unter Austritt von Wasser zu Stande gekommene Verbindung von Oxychinolin und Glukuronsäure vorliegt. Zur Sicherstellung war die Spaltung und Indentificirung der Spaltungsprodukte nothwendig. $\mathrm{Zu}$ dem Zwecke kochte ich $5 \mathrm{~g}$ der freien Säure mit etwa $100 \mathrm{ccm}$. $5 \%$ iger Schwefelsäure mehrere Stunden am Rückflusskühler. Die Anfangs farblose Flüssigkeit färbte sich allmählich gelb bis braun. Beim Neutralisiren trübte sich das Reactionsgemisch und erstarrte zu einem dünnen Krystallbrei. Beim Destilliren dieser trüben Flüssigkeit im Wasserdampfstrom schieden sich sowohl im Kühlrohr als in der Vorlage nadel- 
förmige Krystalle ab. Beim Schütteln des Inhalts der Vorlage mit Chlloroform gingen diese Krystalle in Lösung. Der beim Verdunsten des Chloroforms hinterbleibende Rückstand wurde in verdünntem Alkohol aufgenommen. Es schieden sich lange prismatische glasglänzende Nadeln ab. Krystallform, Schmelzpunkt, der bei $74^{\circ}$ lag, und Löslichkeitsverhältnisse waren diejenigen des o-Oxychinolins. Auch die Stickstoffbestimmung stimmte.

0,1141 g Substanz verbrauchten 8,1 ccm. / 10 N.-Säure. Gefunden: Berechnet für $\mathrm{C}_{9} \mathrm{H}_{7} \mathrm{NO}$ : $9,93 \% \mathrm{~N}$ $9,65 \% \mathrm{~N}$.

Desgleichen zeigten die von mir hergestellten Verbindungen mit Pikrinsäure, Platinchlorid und Brom die für die entsprechenden Verbindungen des o-Oxychinolins charakteristischen Eigenschaften und die geforderte Zusammensetzung. Das Pikrat, schöne gelbe Prismen, schmolz genau bei $203^{\circ}$; das Platindoppelsalz, in kaltem Wasser kaum lösliche, feine hellgelbe Nadeln, enthielt $26,34 \%$ Pt $(0,1003 \mathrm{~g}$ Substanz hinterliessen beim Glühen $0,0264 \mathrm{~g} \mathrm{Pt}$ ) statt der berechneten $26,4 \%$; die Bibromverbindung, in Wasser fast unlösliche, in Benzol lösliche Nadeln, zeigte den richtigen Schmelzpunkt (bei 1930) und enthielt 52,62\% $\mathrm{Br}(0,2447 \mathrm{~g}$ Substanz lieferten 0,3026 g $\mathrm{Br} \mathrm{Ag})$ statt der berechneten $52,8 \%$.

Mehr Mühe erforderte die Isolirung der Glukuronsäure. $\mathrm{Da}$ es von vornherein schwierig, ja unmöglich erscheinen musste, sie von noch ungespaltener Substanz abzutrennen, prüfte ich in einem Vorversuch, wie lange Zeit nöthig war, um die Spaltung $z u$ vollenden. Ich kochte $4 \mathrm{~g}$ Substanz mit $5 \%$ iger Schwefelsäure 10 Stunden am Rückflusskühler. Nach dieser Zeit war der Process noch nicht beendet, denn entfernte ich aus der alkalisch gemachten Flüssigkeit das Oxychinolin durch Ausschütteln mit Chloroform, stellte durch $\mathrm{Zu}-$ satz von Schwefelsäure den ursprünglichen Säuregrad wieder her und kochte weiter, so liess sich nach einer weiteren Stunde abermals freigewordenes Oxychinolin isoliren. Erst nach zwölfstündigem Kochen war die Spaltung eine vollständige. Nach dem Ergebniss dieses Vorversuchs kochte ich $5 \mathrm{~g}$ der Substanz 12 Stunden mit 5\% iger Schwefelsäure am Rückflusskühler. 
Nach der Filtration von ausgeschiedenen Huminsubstanzen wurde mit Barytwasser ganz schwach alkalisch gemacht, mit Chloroform ausgeschüttelt und nun vorsichtig mit Schwefelsäure der überschüssige Baryt ausgefällt. Die vom Baryumsulfat filtrirte Flüssigkeit, welche nichts weiter als Glukuronsäure enthalten konnte, wurde eingedampft, zeigte aber keine Neigung, zu krystallisiren. Ich löste deshalb den Syrup in Wasser, fügte verdünnte Kalilauge bis zur schwach sauren Reaction hinzu und engte wieder ein. Jetzt schieden sich nach einiger Zeit farblose Krystalle ab, welche die Eigenschaften und die Zusammensetzung des glukuronsauren Kali hatten. Die wässerige Lösung reducirte Fehling'sche Flüssigkeit und zeigte Rechtsdrehung.

1. $0,1610 \mathrm{~g}$ Substanz lieferten $0,1828 \mathrm{~g} \mathrm{CO}_{2}$ und $0,0581 \mathrm{~g} \mathrm{H}_{2} 0$.

2. $0,1019 \mathrm{~g}$ Substanz lieferten $0,0379 \mathrm{~g} \mathrm{~K}_{2} \mathrm{SO}_{4}$.

$\begin{array}{rc}\text { Gefunden: } & \text { berechnet für } \mathrm{C}_{6} \mathrm{H}_{9} \mathrm{O}_{7} \mathrm{~K} \\ 30,96 \% \mathrm{C} & 31,03 \% \mathrm{C} \\ 4,01 \% \mathrm{H} & 3,87 \% \mathrm{H} \\ 16,66 \% \mathrm{~K} & 16,81 \% \mathrm{~K} .\end{array}$

Somit ist die nach Eingabe von Chinosol im Harn von Hunden und Kaninchen auftretende Substanz als o-Oxychinolinglukuronsäure charakterisirt. Noch eine andere Veränderung tritt im Harn nach Chinosolzufuhr auf. Diese betrifft die Schwefelsäure, deren Menge in erheblichem Maasse vermehrt ist und zwar gilt das sowohl für die sogenannte freie als auch für die gepaarte Schwefelsäure, wie durch eine Reihe von Versuchen von mir festgestellt wurde. ${ }^{1}$ ) Es kann nicht zweifelhaft sein, dass die Zunahme der letzteren durch die Ausscheidung von Oxychinolinschwefelsäure bedingt ist.

\section{Chinosol.}

Das Auftreten von Oxychinolinglukuronsäure im Harn zwingt zu der Annahme, dass im Organismus aus dem Chinosol Oxychinolin frei geworden ist. Wie schon oben erwähnt, bezeichnet die Fabrik das Chinosol sowohl als oxychinolinsulfo-

1) Ausgedehnte Versuchsreihen nach dieser Richtung sind inzwischen von E. R ost (Arbeiten aus dem Kaiserl. Gesundheitsamt 15, 288) veröffentlicht worden. 
saures, als auch als chinophenylschwefelsaures Kalium. Ueber das Verhalten der aromatischen Sulfosäuren im thierischen Körper liegen nur wenig Beobachtungen vor. So weit mir bekannt sind nur m- und p-phenolsulfosaures Natrium und benzolsulfosaures Natrium in dieser Beziehung geprüft worden und zwar von Salkowski ${ }^{1}$ ) und von Rabuteau. ${ }^{2}$ ) Beide geben übereinstimmend an, dass diese Salze unverändert im Harn erscheinen. Dasselbe gilt von den aetherschwefelsauren Salzen der aromatischen und auch der Fettreihe, wenigstens fand Salkowski dem Hunde eingegebenes aethylschwefelsaures Natrium quantitativ im Harne wieder. Unter diesen Verhältnissen musste es sehr überraschen, dass das Chinosol nicht ebenfalls unverändert den Organismus durchläuft. Diese auffallende Erscheinung sollte bald ihre Erklärung finden. Eine nähere Prüfung des Chinosols ergab nämlich, dass es durchaus keine Aetherschwefelsäure oder Sulfosäure ist, vielmehr ein einfaches Gemenge von o. Oxychinolinsulfat mit Kaliumsulfat darstellt. ${ }^{3}$ ) Folgende Beobachtungen lassen keinen Zweifel darüber. 1. Während Sulfosäuren und Aetherschwefelsäuren gegen Alkali sehr widerstandsfähige Verbindungen sind, gelingt es, aus einer wässerigen Chinosollösung schon nach Zusatz eines schwachen Alkali (Natriumcarbonat oder Natriumbicarbonat) mittelst Chloroform Oxychinolin abzutrennen. 2. Sulfosäuren und Aetherschwefelsäuren werden durch Chlorbaryum nicht gefällt, in einer wässerigen Chinosollösung dagegen entsteht auf Zusatz von Chlorbaryum ein reichlicher Niederschlag von Baryumsulfat, welcher, wie ein quantitativer Versuch zeigte, die gesammte in dem Chinosol enthaltene Schwefelmenge enthält. $0,1392 \mathrm{~g}$ Chinosol lieferten $0,1182 \mathrm{~g} \mathrm{BaSO}_{4}$, entsprechend 12,4\% $\mathrm{S}$, während die von der Fabrik angegebene Chinosolformel 12,2\% S verlangt. 3. Wäscht man Chinosol auf einem Filter mit heissem oder mit einer grösseren Menge kalten absoluten Alkohols, so hinterbleibt ein weisses Krystall-

1) Pflüger's Arch. 4, 91.

2) Gaz. médic. 1881, 115 referiert in Maly's Jahresb. 1881, 195 .

3) $\mathrm{Zu}$ demselben Resultat ist auch Sonntag gekommen, siehe bei Rost a. a. 0. S. 299. 
mehl, das aus Kaliumsulfat besteht. Aus dem alkoholischen Filtrat scheidet sich beim Verdunsten Oxychinolinsulfat in schönen Nadeln aus. 4. Lässt man eine concentrirte Chinosollösung auf dem Uhrglas verdunsten, so erkennt man unter dem Mikroskop sofort Kaliumsulfatkrystalle.

In dem Chinosol ist also Oxychinolin als schwefelsaures Salz in den Organismus eingeführt und dieses vereinigt sich nach Analogie anderer Stoffe zum Theil mit Glukuronsäure, zum Theil mit Schwefelsäure und erscheint in Form dieser beiden gepaarten Verbindungen im Harn. Wie nicht anders zu erwarten, gelang es, dieselbe Oxychinolinglukuronsäure aus dem Harn von Hunden und Kaninchen, die das aus dem Chinosol isolirte o. Oxychinolin*) erhalten hatten, zu gewinnen.

\section{Ueber die Bildung gepaarter Glukuronsäuren im Körper.}

Im Anschluss an das Mitgetheilte sei es mir gestattet, kurz über einen Versuch zu berichten, den ich anstellte, um. Aufschluss über den Bildungsmodus der gepaarten Glukuronsäuren zu erhalten. Er fiel zwar negativ aus, dürfte aber doch von einigem Interesse sein.

Die Glukuronsäure ist ein Oxydationsprodukt des Traubenzuckers, wie durch ihre Verwandlung in Zuckersäure') und durch die Untersuchungen von E. Fischer und Piloty, ${ }^{2}$ ) welche sie aus Zuckerlaktonsäure durch Reduction mit Natriumamalgam erhielten, bewiesen ist. Da die gepaarten Glukuronsäuren im Allgemeinen Fehling'sche Lösung nicht reduciren, so muss die Aldehydgruppe der Glukuronsäure festgelegt sein und es liegt am nächsten, für diese Verbindungen eine den Glukosiden entsprechende Constitution anzunehmen. ${ }^{3}$ ) Der o-0xychinolinglukuronsäure würde also folgende Structurformel zukommen:

*) Ueber das Verhalten der isomeren Oxychinoline im Organismus sind Untersuchungen im hiesigen Laboratorium im Gange.

1) Thierfelder, Diese Zeitschr., XI, 401.

2) Ber. d. deutsch. chem. Gesellsch., 24, 522.

3) Ebenda 26, 2403 u. 2405. 


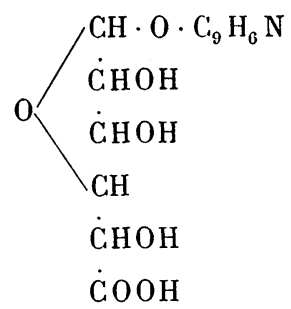

Ueber die Bildung der gepaarten Glukuronsäuren im Thierkörper ist noch nichts Sicheres bekannt. Schmiedeberg und Meyer, ${ }^{1}$ ) welche in der Camphoglukuronsäure die erste Verbindung dieser Art kennen lehrten und die Glukuronsäure zum ersten Mal darstellten, sprechen die Vermuthung aus, dass die Glukuronsäure ein normales Oxydationsprodukt des Traubenzuckers sei, welches für gewöhnlich sofort weiter zerfällt; in dem Fall aber, dass bestimmte Stoffe in den Organismus eingeführt werden, sich mit diesen verbindet und dadurch vor weiterer Verbrennung geschützt wird. Gegen diese Vorstellung wenden sich Fischer und Piloty,$^{2}$ ) indem sie darauf hinweisen, wie unwahrscheinlich es sei, dass bei der Oxydation des Traubenzuckers die Aldehydgruppe zunächst unverändert bleibe, während die endständige Alkoholgruppe in Carboxyl übergehe. Sie sind der Ansicht, dass beim Durchgang der die Bildung gepaarter Glukuronsäuren veranlassenden Substanzen durch den Thierkörper zunächst eine Verbindung derselben oder ihrer Umwandlungsprodukte mit Traubenzucker entsteht, in welcher die Aldehydgruppe des letzteren festgelegt und vor weiterem Angriff geschützt ist, und dass dieses Zwischenprodukt durch Oxydation in die gepaarte Glukuronsäure übergeht. Dieselbe Anschauung über die Entstehung dieser gepaarten Verbindungen ist schon früher von Sundvik ${ }^{3}$ ) ausgesprochen worden. Eine Stütze für diese Hypothese konnte vielleicht das Verhalten der Glukoside im Thierkörper liefern. Erfahren

1) Diese Zeitschr.. III, 422.

2) a. a. 0, S. 524 .

3) Akademische Abhandlung, Helsingfors 1886, referirt in Maly's Jahresbericht, 1886, 76 u. in den Ber. der deutsch. chem. Gesellsch., 19. Referatbd. 762. 
diese eine Oxydation der endständigen Alkoholgruppe zu Carboxyl und werden sie als die entsprechenden gepaarten Glukuronsäuren ausgeschieden, so spricht das entschieden zu Gunsten der Auffassung der oben genannten Forscher, während ein anderes Verhalten allerdings nicht im entgegengesetzten Sinn verwandt werden kann. Die vorliegenden Untersuchungen ${ }^{1}$ ) über das Schicksal natürlicher Glukoside im Organismus geben auf unsere Frage keine bestimmte Antwort. Ich benutzte für meine Versuche das von E. Fischer ${ }^{2}$ ) vor einigen Jahren synthetisch hergestellte a-Methylglukosid. Als Versuchsthiere dienten Kaninchen und Hunde. Der nach Eingabe der Substanz in den Magen gelassene Harn drehte die Polarisationsebene nach rechts und reducirte nach dem Kochen mit Säuren F ehling'sche Lösung, ein Beweis, dass eine völlige Zerstörung des Glukosids nicht stattgefunden hatte; dass das Glukosid selbst oder ein ihm nahestehendes Derivat ausgeschieden war. Alle Versuche, eine gepaarte Glukuronsäure (Methylalkoholglukuronsäure) aus dem Harn zu isoliren, schlugen fehl. Um völlige Gewissheit über ihre An- oder Abwesenheit zu erhalten, versetzte ich den Harn $(60 \mathrm{ccm}$.) eines Kaninchens, welches $2 \mathrm{~g}$ Glukosid bekommen hatte, mit soviel Salzsäure, dass er $5 \% \mathrm{HCl}$ enthielt, erhitzte im kochenden Wasserbad 3 Stunden, entfernte die Salzsäure, machte mit Essigsäure schwach sauer und fügte Hefe hinzu. Es trat lebhafte Gährung ein, nach deren Auf hören die Flüssigkeit $\mathrm{Fe}$ hling'sche Lösung nur noch in der schwachen, für normalen Kaninchenharn typischen Weise reducirte. Damit ist der Beweis geliefert, dass der Harn keine Methylalkoholglukuronsäure enthielt; denn würde sie vorhanden gewesen sein, so hätte nach der Spaltung und Vergährung F'ehling'sche Lösung eine starke Reduction erfahren müssen.

Dieser Versuch schien mir der Mittheilung werth, wenn auch für die Entscheidung der Frage, deretwegen er angestellt wurde, nichts gewonnen worden ist.

1) Vgl. besonders Grisson, Ueber das Verhalten der Glukoside im Thierkörper. Dissert., Rostock 1887.

2) Ber. der deutsch. chem. Gesellsch., 26, 2400. 